\title{
Compromiso y comportamiento ecológico en estudiantes universitarios de Lima y Huaraz
}

Commitment and ecological behavior in university students in Lima and Huaraz

Lupe Garcia $A^{1}$., Oswaldo Orellana M., Manuel Miluanovich C., Elisa Yanac R., Edgar Herrera F., Marco

Espinoza M., Lilia Campos C., Angela Borja G., Angelica Pizarro, Daphne Orellana G., Paul Fernandini

Universidad Nacional Mayor de San Marcos, Lima, Perú

(RECIBIDO 25/09/2015, AcEPTADO 02/12/2015)

\section{RESUMEN}

La presente investigación se orientó a conocer y comparar el compromiso y el comportamiento ecológico y sus factores en estudiantes universitarios de dos universidades públicas de Lima y Huaraz, esta última afectada en una de sus mayores riquezas hídricas (el nevado de Pastoruri). El diseño de investigación es descriptivo-comparativo, la muestra fue conformada por 854 alumnos, de los cuales 315 pertenecen a una universidad pública de Lima y 539 a la universidad pública de Huaraz; 339 son varones y 515 mujeres; los instrumentos de recolección de datos son el cuestionario valorativo de compromiso ecológico, basado en la escala EAKS adaptado por Martínez, F. (2005) y la escala de comportamiento ecológico (ECE), basada en la escala de comportamiento ambiental de Karp y adaptada en Brasil por Pato, 2004; se realizó la adaptación lingüística y el análisis psicométrico de ambos instrumentos, en los dos casos se mantuvieron los mismos factores, lo que varió fue el número total de ítems y la redacción de otros sin variar el contenido; los resultados establecen diferencias significativas en todos los factores de compromiso ecológico en los estudiantes de Lima y Huaraz, en cuanto a sexo se encontraron diferencias significativas solo en el factor de compromiso ecológico afectivo y real, considerando la autodefinición ideológica no se encontraron diferencias en ninguno de los factores. En relación con el comportamiento ecológico en la comparación de ambas universidades se encontraron diferencias significativas en activismo, ahorro de energía y reciclaje; considerando el sexo se encontraron diferencias en los factores de ahorro de agua, energía y reciclaje, y en cuanto a la autodefinición ideológica se encontraron diferencias significativas en el factor de activismo, ahorro de energía y reciclaje.

Palabras clave: Compromiso ecológico, comportamiento ecológico, compromiso ecológico afectivo, compromiso ecológico verbal, compromiso ecológico real.

\section{ABSTRACT}

This research was oriented to know and compare the commitment, ecological behaviour and their factors in university students from two public universities in Lima and Huaraz, the latter affected in one of its most important hydric resources (Pastoruri snow-capped mountain).

1 Docente Principal de la Facultad de Psicología de la UNMSM, E-mail: Igarciape@yahoo.es 
The design of the research project is descriptive-comparative, the sample was represented by 854 students, 315 are from a public university in Lima and 539 are from a public university in Huaraz; 339 are men and 515 are women, the collection instruments to collect data are the value questionnaire of ecological commitment, based on the EAKS scale adapted by Martinez, F. (2005) and the ecological behaviour scale (ECE), based on Karp's environmental behaviour scale and adapted in Brazil by Pato, 2004; Linguistic adaptation and psychometric analysis of both instruments were realized, in both cases, the same factors remained intact, what varied was the total number of items and the writing of others without affecting content; the results establish meaningful differences in all the factors of ecological commitment in the students from Lima and Huaraz, regarding sex, we found meaningful differences only in the factor of affective and ecological real commitment, taking into consideration the ideological autodefinition, we did not find any differences in the factors. Regarding the ecological behaviour in the comparison of the universities of both cities, we found meaningful differences in activism, energy saving and recycling, regarding sex, we found differences in the factors of water and energy saving and recycling, and regarding the ideological autodefinition, we found meaningful differences in the factor of activism, energy saving and recycling.

Keywords: Commitment ecological, ecological behaviour environmental performance, environmental commitment affective, verbal ecological commitment, real ecological commitment.

\section{INTRODUCCIÓN}

La presente investigación partió del reconocimiento que uno de los problemas por los que atraviesa la humanidad es el desequilibrio climático y la afectación a la integridad ecológica, en gran parte producida por la acción de las personas independientemente de su grado de instrucción; quienes realizan prácticas destructivas contra el medio ambiente o son pasivos e indiferentes frente a estos hechos.

De parte de las universidades no existen propuestas que se expresen en políticas universitarias orientadas a la conservación del medioambiente y si existen son declaraciones o propuestas que no se expresan en acciones, por ello nuestro interés en investigar esta temática en los estudiantes, buscando analizar el compromiso y comportamiento ecológico en universitarios de Lima y Huaraz; esta última afectada en una de sus mayores riquezas hídricas (el nevado de Pastoruri). Entiéndase que el compromiso ecológico es más bien una disponibilidad psicológica favorable respecto al medioambiente, en su cuidado y preservación, y la conducta es el conjunto de acciones realizadas por las personas en su relación directa o indirecta con él.

También interesa analizar si el sexo y la autodefinición ideológica son variables que establecen diferencias en el compromiso y comportamiento ecológico, dado que aún persiste una socialización diferenciada en hombres y mujeres y de otro lado hay ideologías más proclives a la preservación del medioambiente.

A nivel internacional diferentes investigadores han abordado las problemáticas medioambientales, entre los cuales tenemos:

Corral,V. y Tapia, C. (2013) investigaron las virtudes de la humanidad, justicia y moderación y su relación con la conducta sustentable, en 400 personas de la 
Lupe García, Oswaldo Orellana, Manuel Miljanovich, Elisa Yanac, Edgar Herrera, Marco Espinoza, Lilia Campos,

Angela Borja, Angelica Pizarro, Daphne Orellana, Paul Fernandin

ciudad de México, encontraron una correlación entre las virtudes de justicia y moderación y las conductas sustentables; que se manifiestan en actos pro-ecológicos, altruistas y equitativos. La menor correlación se logró con los valores vinculados a la humanidad y la conducta sustentable.

Fraijo, B., Corral, V.,Tapia,C. y García, F. (2012) construyeron una escala de orientación hacia la sustentabilidad para niños de educación básica, basada en la escala para universitarios, el objetivo fue probar la pertinencia de la escala de orientación a la sustentabilidad en niños, se aplicó a una muestra de 200 niños de $6^{\circ}$ grado de educación básica en la ciudad de Hermosillo y se encontró que adaptado a la infancia era un buen instrumento para evaluar la orientación hacia la sustentabilidad.

Moyano, E., Cornejo, F. y Gallardo, I. (2011) investigaron las creencias y conductas ambientales, el liberalismo económico y la felicidad, bajo el nuevo paradigma ambiental, en una muestra de 409 jóvenes urbanos y rurales de una región de Chile, y encontraron que la procedencia urbana y rural no tiene relación con ninguna de las variables y las creencias positivas a favor del medioambiente están relacionadas positivamente con la conducta pro-ambiental y la felicidad, no así con el liberalismo económico, asimismo no se encontraron diferencias de género.

Palavecinos, M. y Jaime, A. (2010) investigaron la preocupación y conducta ecológica responsable en estudiantes universitarios, y compararon las respuestas de estudiantes chilenos y españoles, se encontraron diferencias que fueron analizadas en función de variables culturales, psicosociales y la formación universitaria recibida en ambos, y encontraron diferencias significativas tanto en la conducta como en la preocupación ecológica.

Corral, V., Tapia, C., Frías, M., Fraijo, B. y Gonzales, D. (2009) investigaron la orientación a la sostenibilidad como base para el comportamiento pro-social y pro-ecológico, en la que se planteó un modelo de "orientación a la sostenibilidad" que incluye un conjunto de factores psicológicos como el altruismo, la austeridad, las conductas proecológicas, la autopresentación, la afinidad por la diversidad, el aprecio por lo natural, los sentimientos de indignación por el daño ambiental, finalmente concluyeron que todas estas dimensiones correlacionan entre sí.

Calvo, A. y Aguilar, M. (2008) investigaron el comportamiento ecológico responsable, estableciendo la relación entre los valores y determinados comportamientos ecológicamente responsables y a su vez se exploró la contribución de los valores en la explicación de cada conducta concreta. Se analizaron los valores biosféricos, los valores altruistas y egocéntricos, los resultados resaltan la importancia de los valores biosféricos en la explicación del comportamiento ecológicamente responsable.

Martínez, F., Martínez, J. y Gazquez, L. (2005) investigaron las actitudes y comportamientos ambientales elementos determinantes en el consumo de alimentos ecológicos, se analizó las características de las actitudes y conducta de los sujetos estableciendo el perfil de los consumidores de alimentos ecológicos, se exploró 
la capacidad predictiva de las variables relacionadas con las actitudes respecto a conductas ecológicas y se elaboró un instrumento de valoración del compromiso ambiental.

Moreno, M., Corraliza, J. y Ruiz, J. (2005) se propusieron elaborar una escala de actitudes ambientales hacia problemas específicos que finalmente quedó con 50 ítems, a su vez establecieron los factores de preocupación individual, preocupación social, confianza y criterio. Este instrumento se aplicó a 1,433 estudiantes españoles, en el que se encontró que el transporte es una de las problemáticas considerada como más importante en la muestra madrileña.

Berenger, J., Corraliza,J., Moreno, M. y Rodríguez, L. (2002) investigaron la medida de las actitudes ambientales: Propuesta de una escala de conciencia ambiental, en la cual parten de una posición crítica respecto de las escalas de evaluación ambiental existentes y se plantean desarrollar una escala de actitudes ambientales con tres premisas: la primera, considerar la relevancia de los temas en el comportamiento ambiental; la segunda, definir una medida que establezca la especificidad de los comportamientos; y tercero, establecer los contenidos de la evaluación en actitudes ambientales a nivel personal y contextual. Con ello se concluye en la importancia de la evaluación multidimensional y específica, para efectos de explicación e intervención, finalmente elaboraron una escala de conciencia ambiental.

Los objetivos planteados fueron:

\section{Objetivo general}

Describir y comparar el compromiso y comportamiento ecológico de estudiantes universitarios de dos universidades públicas de Lima y Huaraz.

\section{Objetivos específicos}

Establecer las diferencias en el compromiso ecológico considerando los factores: compromiso ecológico afectivo, compromiso ecológico real y compromiso ecológico verbal de estudiantes universitarios de dos universidades públicas de Lima y Huaraz.

Establecer las diferencias en el comportamiento ecológico considerando los factores activismo, ahorro de agua y energía, limpieza urbana y reciclaje en estudiantes universitarios de dos universidades públicas de Lima y Huaraz.

Establecer las diferencias según sexo en el compromiso y comportamiento ecológico de estudiantes universitarios de dos universidades públicas de Lima y Huaraz.

Establecer las diferencias en el compromiso y comportamiento ecológico de estudiantes universitarios en función de la autodefinición ideológica. 
Lupe García, Oswaldo Orellana, Manuel Miljanovich, Elisa Yanac, Edgar Herrera, Marco Espinoza, Lilia Campos, Angela Borja, Angelica Pizarro, Daphne Orellana, Paul Fernandin

\section{Hipótesis general}

Existen diferencias significativas en el compromiso y comportamiento ecológico de estudiantes universitarios de dos universidades públicas de Lima y Huaraz.

\section{Hipótesis específicas}

Existen diferencias significativas en el compromiso ecológico afectivo, verbal y real en estudiantes universitarios de dos universidades públicas de Lima y Huaraz.

Existen diferencias significativas en el comportamiento ecológico expresado en la dimensión de activismo, ahorro de agua y energía, limpieza urbana y reciclaje de estudiantes universitarios de Lima y Huaraz.

Existen diferencias significativas en los factores del compromiso y comportamiento ecológico de estudiantes universitarios de acuerdo al sexo.

Existen diferencias significativas en el compromiso y comportamiento ecológico de estudiantes universitarios según autodefinición ideológica.

\section{MÉTODO}

\section{Diseño}

El diseño utilizado es descriptivo-comparativo, porque busca identificar las diferencias y similitudes en el compromiso y comportamiento ecológico de estudiantes universitarios de Lima y Huaraz, de diferente sexo y con autodefiniciones ideológicas distintas.

\section{Participantes}

La muestra estuvo constituida por 854 alumnos, de los cuales, 315 corresponden a la universidad pública de Lima y 539 corresponden a la universidad pública de Huaraz; 339 son varones y 515 mujeres. Respecto a la autodefinición ideológica 72 se definen de derecha, 49 de centro derecha, 96 de centro, 87 de centro izquierda, 38 de izquierda y 512 consideran que no tienen ninguna orientación ideológica.

\section{Instrumentos}

Los instrumentos utilizados fueron la escala de comportamiento ecológico y el cuestionario de compromiso ecológico, ambos instrumentos fueron adaptados y sometidos al análisis psicométrico; en el caso de la escala de comportamiento ecológico se utilizó la versión adaptada por Pato (2004) basada a su vez en la escala de comportamiento ecológico de Karp (1996). La escala final mantuvo los 4 factores o dimensiones: Activismo, limpieza urbana, ahorro de energía y reciclaje; después del análisis psicométrico se eliminaron tres ítems quedando con un total 
de 23; el cuestionario valorativo de compromiso ecológico, basado en la escala EAKS adaptado por Martínez, F. (2005) fue a su vez adaptado por el equipo de investigación modificando la redacción de los ítems 5,10,12, 13 y 14; pero se mantuvieron los 18 ítems y los mismos factores: compromiso ecológico afectivo, verbal y real. El compromiso verbal se define como la intención positiva o negativa de comportarse de forma respetuosa con el medio ambiente, el compromiso afectivo como la disposición emocional positiva o negativa respecto al medioambiente y el compromiso real que es la conducta o comportamiento medioambiental

\section{RESULTADOS}

En la tabla 1 se presentan los resultados de las medias y desviaciones de los puntajes obtenidos en el cuestionario de compromiso ecológico por los estudiantes de Lima y Huaraz, en la que se observa que existen diferencias en los puntajes medios de los estudiantes de ambas ciudades, siendo los estudiantes de Huaraz los que obtienen una mayor puntuación.

Tabla 1. Compromiso ecológico según la ciudad de procedencia.

\begin{tabular}{lcccc}
\cline { 2 - 5 } & Ciudad & N. $^{\mathbf{0}}$ & Media & DT \\
\hline $\begin{array}{l}\text { Compromiso Ecológico } \\
\text { Afectivo }\end{array}$ & Lima & 315 & 22.30 & 2.89 \\
\cline { 2 - 5 } & Huaraz & 539 & 23.27 & 2.70 \\
\hline $\begin{array}{l}\text { Compromiso Ecológico } \\
\text { Verbal }\end{array}$ & Lima & 315 & 15.47 & 2.07 \\
\cline { 2 - 5 } $\begin{array}{l}\text { Compromiso Ecológico } \\
\text { Real }\end{array}$ & Huaraz & 539 & 15.72 & 2.04 \\
\hline \multirow{2}{*}{$\begin{array}{l}\text { Total Compromiso } \\
\text { Ecológico }\end{array}$} & Lima & 315 & 14.93 & 2.74 \\
\cline { 2 - 5 } & Huaraz & 539 & 16.70 & 2.98 \\
\hline
\end{tabular}

En la tabla 2 se consigna los resultados de las medias y desviaciones de comportamiento ecológico comparando las universidades de las dos ciudades, por lo que podemos observar que existe una ligera diferencia entre los puntajes medios de los estudiantes de las dos ciudades, siendo la muestra de Huaraz la que posee mayor puntuación en el puntaje global y en las dimensiones de activismo y reciclaje; mientras que Lima posee mayor puntuación en lo referente al ahorro de energía y limpieza urbana. 
Lupe García, Oswaldo Orellana, Manuel Miljanovich, Elisa Yanac, Edgar Herrera, Marco Espinoza, Lilia Campos, Angela Borja, Angelica Pizarro, Daphne Orellana, Paul Fernandin

Tabla 2. Comportamiento ecológico según la ciudad de procedencia.

\begin{tabular}{|c|c|c|c|c|}
\hline & & N. ${ }^{\circ}$ & Media & DT \\
\hline \multirow{2}{*}{ Activismo } & Lima & 315 & 10.73 & 2.47 \\
\hline & Huaraz & 539 & 12.46 & 2.51 \\
\hline \multirow{2}{*}{ Ahorro de Energía } & Lima & 315 & 24.05 & 3.27 \\
\hline & Huaraz & 539 & 23.01 & 3.57 \\
\hline \multirow{2}{*}{ Limpieza Urbana } & Lima & 315 & 15.86 & 2.50 \\
\hline & Huaraz & 539 & 15.73 & 2.63 \\
\hline \multirow{2}{*}{ Reciclaje } & Lima & 315 & 16.46 & 3.22 \\
\hline & Huaraz & 539 & 16.91 & 3.69 \\
\hline \multirow{2}{*}{ Total Conducta Ecológica } & Lima & 315 & 67.10 & 7.88 \\
\hline & Huaraz & 539 & 68.11 & 9.31 \\
\hline
\end{tabular}

En la tabla 3 se aprecia las diferencias existentes en cada uno de los factores y compromiso ecológico global, según la ciudad de procedencia; es así que se puede identificar que existen diferencias significativas a un nivel de confianza del 99\% en las dimensiones de compromiso afectivo y real $(\alpha=0.00 ; \alpha<0.01)$ del mismo modo que en la puntuación total $(\alpha=0.00 ; \alpha<0.01)$ y en la dimensión de compromiso ecológico verbal también existen diferencias a un nivel de confianza del 95\% $(\alpha=0.05, \alpha=0.05)$.

Tabla 3. Diferencias en las dimensiones de compromiso ecológico según la ciudad de procedencia.

\begin{tabular}{lcccc}
\cline { 2 - 5 } & $\begin{array}{c}\text { U de Mann- } \\
\text { Whitney }\end{array}$ & $\begin{array}{c}\text { W de } \\
\text { Wilcoxon }\end{array}$ & Z & $\begin{array}{c}\text { Sig. asintót. } \\
\text { (bilateral) }\end{array}$ \\
\hline $\begin{array}{l}\text { Compromiso Ecológico } \\
\text { Afectivo }\end{array}$ & 68485.00 & 118255.00 & -4.75 & 0.00 \\
\hline $\begin{array}{l}\text { Compromiso Ecológico } \\
\text { Verbal }\end{array}$ & 78289.50 & 128059.50 & -1.92 & 0.05 \\
\hline $\begin{array}{l}\text { Compromiso Ecológico } \\
\text { Real }\end{array}$ & 54756.00 & 104526.00 & -8.71 & 0.00 \\
\hline $\begin{array}{l}\text { Total Compromiso } \\
\text { Ecológico }\end{array}$ & 60599.50 & 110369.50 & -6.99 & 0.00 \\
\hline
\end{tabular}

En la tabla 4 se observa que existen diferencias significativas en el comportamiento ecológico expresado en la dimensión de activismo, ahorro de energía, a un nivel de significación del 99\% $(\alpha=0.00 ; \alpha<0.01)$ y reciclaje a un nivel de significación del 95\% $(\alpha=0.05 ; \alpha<0.03)$ entre estudiantes universitarios de Lima y Huaraz. 
COMPROMISO Y COMPORTAMIENTO ECOLÓGICO EN ESTUDIANTES UNIVERSITARIOS DE LIMA Y HUARAZ

Tabla 4. Diferencias en comportamiento ecológico y sus dimensiones en Lima y Huaraz.

\begin{tabular}{lcccc} 
& $\begin{array}{c}\text { U de Mann- } \\
\text { Whitney }\end{array}$ & $\begin{array}{c}\text { W de } \\
\text { Wilcoxon }\end{array}$ & $\mathbf{Z}$ & $\begin{array}{c}\text { Sig. asintót. } \\
\text { (bilateral) }\end{array}$ \\
\hline Activismo & 52496.50 & 102266.50 & -9.38 & 0.00 \\
\hline Ahorro de Energía & 69854.50 & 215384.50 & -4.34 & 0.00 \\
\hline Limpieza Urbana & 82548.00 & 228078.00 & -0.68 & 0.50 \\
\hline Reciclaje & 77472.50 & 127242.50 & -2.14 & 0.03 \\
\hline Total Conducta Ecológica & 79358.00 & 129128.00 & -1.59 & 0.11 \\
\hline
\end{tabular}

En la tabla 5 se observa la media obtenida según el sexo de los sujetos en cada una de las dimensiones y en el puntaje total del compromiso ecológico. A nivel global, las medias más altas corresponden a las mujeres, y en cada una de las dimensiones la media más alta corresponde a las mujeres en compromiso afectivo y verbal y los hombres obtienen una media más alta en la dimensión de compromiso real.

Tabla 5. Compromiso ecológico según sexo de los evaluados.

\begin{tabular}{lllll}
\cline { 2 - 4 } & Sexo & N & Media & DT \\
\hline $\begin{array}{l}\text { Compromiso Ecológico } \\
\text { Afectivo }\end{array}$ & Varones & 339 & 22.63 & 2.89 \\
\cline { 2 - 5 } $\begin{array}{l}\text { Compromiso Ecológico } \\
\text { Verbal }\end{array}$ & Mujeres & 515 & 23.10 & 2.74 \\
\cline { 2 - 5 } $\begin{array}{l}\text { Compromiso Ecológico } \\
\text { Real }\end{array}$ & Varones & 339 & 15.45 & 2.12 \\
\cline { 2 - 5 } $\begin{array}{l}\text { Total Compromiso } \\
\text { Ecológico }\end{array}$ & Mujeres & 515 & 15.74 & 2.00 \\
\hline
\end{tabular}

En la tabla 6 se puede observar que existen diferencias significativas a un nivel de confianza del $95 \%$ según sexo en la dimensión de compromiso ecológico afectivo $(\alpha=0.02 ; \alpha<0.05)$ y en compromiso ecológico real $(\alpha=0.04 ; \alpha<0.05)$, en la dimensión de compromiso ecológico verbal no existen diferencias. 
Lupe García, Oswaldo Orellana, Manuel Miljanovich, Elisa Yanac, Edgar Herrera, Marco Espinoza, Lilia Campos, Angela Borja, Angelica Pizarro, Daphne Orellana, Paul Fernandin

Tabla 6. Diferencias en compromiso ecológico y sus dimensiones según sexo.

\begin{tabular}{|c|c|c|c|c|}
\hline & $\begin{array}{l}\text { U de Mann- } \\
\text { Whitney }\end{array}$ & $\begin{array}{c}\text { W de } \\
\text { Wilcoxon }\end{array}$ & $\mathbf{Z}$ & $\begin{array}{c}\text { Sig. asintót. } \\
\text { (bilateral) }\end{array}$ \\
\hline $\begin{array}{l}\text { Compromiso Ecológico } \\
\text { Afectivo }\end{array}$ & 79217.50 & 136847.50 & -2.30 & 0.02 \\
\hline $\begin{array}{l}\text { Compromiso Ecológico } \\
\text { Verbal }\end{array}$ & 82921.50 & 140551.50 & -1.25 & 0.21 \\
\hline $\begin{array}{l}\text { Compromiso Ecológico } \\
\text { Real }\end{array}$ & 80098.00 & 212968.00 & -2.05 & 0.04 \\
\hline $\begin{array}{l}\text { Total Compromiso } \\
\text { Ecológico }\end{array}$ & 86886.50 & 144516.50 & -0.12 & 0.91 \\
\hline
\end{tabular}

En la tabla 7 se aprecia la media obtenida según el sexo de los sujetos en cada una de las dimensiones y en el puntaje total del comportamiento ecológico. A nivel global la media es más alta en la muestra de mujeres. Solo en la dimensión de activismo los hombres obtienen una media más alta.

Tabla 7. Comportamiento ecológico según el sexo de los evaluados.

\begin{tabular}{lcccc} 
& Sexo & N & Media & DT \\
\hline \multirow{2}{*}{ Activismo } & Varones & 339 & 12.02 & 2.63 \\
\cline { 2 - 5 } Ahorro de Energía & Mujeres & 515 & 11.69 & 2.62 \\
\cline { 2 - 5 } & Varones & 339 & 22.71 & 3.84 \\
\cline { 2 - 5 } Limpieza Urbana & Mujeres & 515 & 23.85 & 3.17 \\
\cline { 2 - 5 } & Varones & 339 & 15.62 & 2.64 \\
\cline { 2 - 5 } Reciclaje & Mujeres & 515 & 15.88 & 2.54 \\
\hline \multirow{2}{*}{$\begin{array}{l}\text { Total Conducta } \\
\text { Ecológica }\end{array}$} & Varones & 339 & 16.14 & 3.70 \\
\cline { 2 - 5 } & Mujeres & 515 & 17.14 & 3.35 \\
\hline
\end{tabular}

En la tabla 8 se aprecia que existen diferencias significativas a un nivel de confianza del $99 \%$ en las dimensiones de ahorro de energía, reciclaje y en el total de conducta ecológica $(\alpha=0.00 ; \alpha<0.01)$ según género.

En ambas dimensiones: ahorro de energía y reciclaje, así como en el puntaje total la diferencia es a favor de las mujeres. 
Tabla 8. Diferencia de medias en el comportamiento ecológico según el sexo de los sujetos.

\begin{tabular}{lcccc}
\cline { 2 - 5 } & $\begin{array}{c}\text { U de Mann- } \\
\text { Whitney }\end{array}$ & $\begin{array}{c}\text { W de } \\
\text { Wilcoxon }\end{array}$ & $\mathbf{Z}$ & $\begin{array}{c}\text { Sig. asintót. } \\
\text { (bilateral) }\end{array}$ \\
\hline Activismo & 81019.50 & 213889.50 & -1.79 & 0.07 \\
\hline Ahorro de Energía & 72730.00 & 130360.00 & -4.15 & 0.00 \\
\hline Limpieza Urbana & 82702.00 & 140332.00 & -1.31 & 0.19 \\
\hline Reciclaje & 72928.00 & 130558.00 & -4.09 & 0.00 \\
\hline Total Conducta Ecológica & 75513.50 & 133143.50 & -3.34 & 0.00 \\
\hline
\end{tabular}

En la tabla 9 en base a la prueba de Kruskal Wallis se puede identificar que no existen diferencias significativas en ninguna de las dimensiones de compromiso ecológico. Según autodefinición ideológica.

En relación al comportamiento ecológico si existen diferencias significativas a un nivel de confianza del $99 \%$ en la dimensión de activismo, ahorro de energía y reciclaje $(\alpha=0.00 ; \alpha<0.01)$; en limpieza urbana y el total de la conducta ecológica no existen diferencias significativas.

Tabla 9. Diferencias en compromiso y comportamiento ecológico según auto-definición ideológica de los estudiantes con la Prueba de Kruskal Wallis.

\begin{tabular}{lccc}
\cline { 2 - 4 } & Chi-cuadrado & gl & Sig. asintót. \\
\hline $\begin{array}{l}\text { Compromiso Ecológico } \\
\text { Afectivo }\end{array}$ & 6.55 & 5 & 0.26 \\
\hline $\begin{array}{l}\text { Compromiso Ecológico } \\
\text { Verbal }\end{array}$ & 10.19 & 5 & 0.07 \\
\hline Compromiso Ecológico Real & 3.41 & 5 & 0.64 \\
\hline Total Compromiso Ecológico & 5.86 & 5 & 0.32 \\
\hline Activismo & 17.01 & 5 & 0.00 \\
\hline Ahorro de Energía & 15.17 & 5 & 0.01 \\
\hline Limpieza Urbana & 4.18 & 5 & 0.52 \\
\hline Reciclaje & 15.17 & 5 & 0.01 \\
\hline Total Conducta Ecológica & 8.95 & 5 & 0.11 \\
\hline
\end{tabular}




\section{DISCUSIÓN}

Desde el punto de vista descriptivo las puntuaciones más altas de los factores de compromiso ecológico son en orden descendente: compromiso ecológico afectivo, compromiso real y compromiso verbal; en cuanto a los niveles en el factor afectivo y real los niveles son altos, en el factor de compromiso verbal obtiene un nivel medio. En comportamiento ecológico las puntuaciones en orden descendiente son: ahorro de energía, reciclaje, limpieza urbana y activismo; y en los niveles altos, las dimensiones ahorro de energía y limpieza urbana; y a nivel medio, el activismo y reciclaje.

Al comparar los resultados totales y de cada una de las dimensiones de compromiso ecológico se encuentra que en todas las dimensiones existen diferencias significativas; en la dimensión afectiva y real las diferencias son al $99 \%$ de nivel de confianza y la dimensión de compromiso real al $95 \%$, todas las diferencias son favorables a los estudiantes de Huaraz, es decir que en ellos es más alto el compromiso ecológico comparado con los estudiantes de Lima; probablemente los daños causados a una de sus mayores riquezas hídricas (Pastoruri) ha desarrollado una disponibilidad positiva para expresar actitudes favorables, resultados similares en cuanto a los factores fueron encontrados por Martínez-Carrasco, Martínez y Gazquez (2007)

En cuanto al comportamiento ecológico se encontraron diferencias en las dimensiones de activismo favorables a los estudiantes de Huaraz, es decir tienen más alta puntuación en las acciones que realizan y que están vinculadas a la preservación del medioambiente. En la dimensión ahorro de energía también existen diferencias significativas al 99\%, en este caso favorables a los estudiantes de Lima, lo que significaría que tienen mayor puntuación en las conductas vinculadas al uso/desperdicio del agua y de energía eléctrica; probablemente los altos costos de los servicios de agua y electricidad en Lima y que afectan directamente a la economía familiar son variables que disuaden de conductas de despilfarro de agua y energía.

En el total de comportamiento ecológico y limpieza urbana no existen diferencias estadísticamente significativas, sin embargo en el primer caso las diferencias sin ser significativas son favorables a la muestra de Huaraz; en limpieza urbana los resultados son favorables a la muestra de Lima, lo que podría ser explicada por una campaña sostenida en los diferentes distritos respecto a la basura y limpieza.

En la comparación por sexo se encontraron diferencias significativas en el factor de compromiso ecológico afectivo favorable a las mujeres, lo que muestra una mayor preocupación y afectación en las mismas, explicable por la socialización femenina, también existen diferencias significativas en la dimensión de compromiso real favorable a los hombres, lo que indicaría están más dispuestos a realizar acciones que favorezcan la preservación del medio y acciones orientadas a incrementar su información al respecto, resultados similares fueron encontrados por Pato, Ros, Tamayo (2005). 
En cuanto a comportamiento ecológico se encontraron diferencias estadísticamente significativas en los factores de ahorro de agua y energía y reciclaje, en ambos casos las diferencias son favorables a las mujeres, lo que estaría indicando que las mujeres practican conductas de ahorro y reaprovechamiento de recursos con mayor frecuencia y que además son las que dentro de sus roles les está asignada dichas actividades. No se encontraron diferencias estadísticamente significativas en el total de comportamiento ecológico y en la dimensión de activismo.

Antes de analizar los resultados según auto-definición ideológica, es interesante señalar que el $60 \%$ de la muestra se auto-define sin ninguna ideología, el $40 \%$ restante está distribuido entre derecha, izquierda y centro. Al analizar las diferencias en el compromiso ecológico según autodefinición ideológica, no se encontraron diferencias significativas ni en el puntaje global ni en cada uno de los factores. En cuanto al comportamiento ecológico se encontraron diferencias significativas en las dimensiones de activismo, ahorro de energía y reciclaje; en activismo y ahorro de energía favorables a los que se auto-definen como de derecha y en reciclaje favorable a quienes no se identifican con ninguna ideología. Estos resultados son interesantes, por cuanto tradicionalmente han sido las personas de orientación izquierdista o de centro izquierda quienes han realizado mayor activismo y disponibilidad a la protección ecológica; pero igualmente nos muestra una realidad, la distancia de los jóvenes respecto a las ideologías.

\section{CONCLUSIONES Y RECOMENDACIONES}

En relación al compromiso ecológico, existen diferencias significativas en todos los factores de compromiso ecológico en los estudiantes universitarios de dos universidades públicas de Lima y Huaraz.

Respecto al comportamiento ecológico, existen diferencias significativas en el factor, ahorro de agua, energía y reciclaje según la universidad de procedencia. No existen diferencias en la dimensión limpieza urbana.

Considerando el sexo, existen diferencias en el factor de compromiso ecológico afectivo y real. No existen diferencias en la dimensión compromiso ecológico verbal y el total de compromiso ecológico.

Existen diferencias significativas en la dimensión ahorro de agua y energía, reciclaje y total de comportamiento ecológico de acuerdo al sexo. No existen diferencias significativas en activismo y limpieza urbana.

Existen diferencias significativas en el factor compromiso ecológico afectivo y real según auto-definición ideológica. En los otros factores de compromiso ecológico verbal no existen diferencias significativas.

Existen diferencias significativas en el factor de activismo, ahorro de agua, energía y reciclaje según auto-definición ideológica. No existen diferencias significativas en la dimensión limpieza urbana según autodefinición ideológica. 
Lupe García, Oswaldo Orellana, Manuel Miljanovich, Elisa Yanac, Edgar Herrera, Marco Espinoza, Lilia Campos, Angela Borja, Angelica Pizarro, Daphne Orellana, Paul Fernandin

Las recomendaciones son:

Propiciar que las universidades realicen programas de sensibilización respecto a la conservación del medio ambiente en toda la comunidad universitaria.

Promover la formulación de políticas de limpieza y el ahorro de agua y energía, al interior de los ambientes universitarios.

Promover investigaciones orientadas a la educación ambiental y la formación de promotores de cuidado del medioambiente.

\section{REFERENCIAS BIBLIOGRÁFICAS}

Berenguer, J., Corraliza, J. (2000) Preocupación ambiental y comportamientos ecológicos, Psicothema, 12(3) 325-329.

Calvo, A., Aguilar, M. (2008) El comportamiento ecológico responsable: un análisis desde los valores biosféricos, sociales, altruistas y egoístas, Rev. Electrónica de investigación y docencia, 1, 11-25

Corral,V., Tapia,C., Ortiz, A., Fraijo, B. (2013) Las virtudes de la humanidad, justicia y moderación y su relación con la conducta sustentable, Rev. Latinoamericana de psicología. 45 (3) 363-374.

Corral, V., Tapia,C., Frias, M., Fraijo, B.,Gonzales, D. (2009) Orientación a la sostenibilidad como base para el comportamiento pro-social y pro-ecológico, Medio ambiente y comportamiento humano. 10 (3) 195-215.

Corral, V. (2012) Sustentabilidad y psicología positiva. México: Manual moderno

Corral, V. (2012) Psicología de la sustentabilidad. México: Trillas

Fraj, A. Martínez, E. (2005) El nivel de conocimiento medioambiental como factor moderador de la relación entre la actitud y el comportamiento ecológico, Investigaciones Europeas de dirección y economía de la empresa, 11 (1) $1135-2523$

Fraijo, B., Corral, V., Tapia, C., García, F. (2012) Adaptación y prueba de una escala de orientación a la sustentabilidad en niños de sexto año de educación básica, Rev. Mexicana de investigación educativa. 17 (55) 1091-1117.

Martínez,F., Martínez,J., Gazquez, L. (2007) Actitudes y comportamientos ambientales elementos determinantes en el consumo de alimentos ecológicos, II congreso de la Asociación Hispano-portuguesa de economía de los recursos naturales y ambientales. Portugal.

Moreno, M., Corraliza, J.Ruiz, J. (2005) Escala de actitudes ambientales hacia problemas específicos, Rev. Psicothema, 17 (3) 502-508. 
Moyano, E., Cornejo, F., Gallardo, I. (2011) Creencias y conductas ambientales, liberalismo económico y felicidad. Acta colombiana de Psicología, 14 (2) 69-77.

Palavecinos, M., Jaime, A. (2010) Preocupación y conducta ecológica responsable en estudiantes universitarios. Congreso Iberoamericano de Educación.

Pato, C, Ros, M, Tamayo, A. (2005) Creencias y comportamiento ecológico: un estudio empírico con estudiantes Brasileños. Medio ambiente y comportamiento humano, 6 (1) 5-22.

Zapata, R., Castrechini, A. (2011) Conducta pro-ambiental y personalidad: análisis de un barrio de Lima. Quaderns de psicología, 13 (1) 47-61. 